\title{
Antimicrobial resistance profile among bacteria isolated from patients presenting with wounds at Kabale Regional Referral Hospital, South western Uganda
}

Andrew Baguma ( $\sim$ abaguma@kab.ac.ug)

Mbarara University of Science and Technology https://orcid.org/0000-0001-8053-4130

Benson Musinguzi

Kampala International University - Western Campus

Atek Atwiine Kagirita

Central Public Health Laboratories Ministry of Health Uganda Joel Bazira

Mbarara University of Science and Technology

Research

Keywords: Bacterial wound infections, Antimicrobial Drug Resistance

Posted Date: March 16th, 2020

DOI: https://doi.org/10.21203/rs.3.rs-17292/v1

License: (c) (1) This work is licensed under a Creative Commons Attribution 4.0 International License.

Read Full License 


\section{Abstract}

Background Bacterial Wound infection and antimicrobial resistance remains a public health challenge. The challenge remains worse due to nosocomial bacterial infection often characterized by multidrug resistance. Infected wounds are often associated with delayed epidermal maturation resulting into prolonged hospitalization. Data on profile of clinical significant bacteria and their respective antibiotic drug resistance in Uganda is still limited. In this study we emphasized on phenotypic characterization of bacteria that cause wound infections at Kabale Regional Hospital (KRRH) and determining the respective antimicrobial susceptibility profiles.

Methods Between June 2016 - to June 2017 a total of 276 Pus specimens were collected from patients at KRRH and analyzed for bacterial infection by standard bacterial cultures techniques. Pus specimens were all from wounds (surgical and non-surgical). Antibiotic susceptibility testing was performed and reported based on CLSI guidelines.

Results One-hundred and ninety-five specimens were positive following bacterial culture $(70.7 \%)$. Staphylococcus aureus and Escherichia coli were the most frequently isolated bacteria. Antibiotic drug resistance testing revealed that $68 \%$ of $\mathrm{S}$. aureus isolates were Methicillin resistant. For Escherichia coli isolates, $73 \%$ were ciprofloxacin and levofloxacin resistant while resistance to Imipenem was common among Klebsiella sp.

Conclusions Wound infection is mainly caused by gram negative bacteria particularly, Escherichia coli , Klebsiella spp., Proteus spp., and Pseudomonas spp. G positive cocci particularly S. aureus is also an important pathogen among other implicated gram positive cocci. There are high levels of multi antimicrobial resistance among both Gram negative and Gram positive bacteria.

\section{Background}

Antimicrobial resistance remains a public health challenge impacting negatively on the quality of health care delivery ${ }^{[1]}$. Bacterial drug resistance has demonstrated prospective ability to spread from one individual to another within and outside healthcare setting ${ }^{2}$. Several factors fueling antimicrobial resistance have been described worldwide, with unregulated access to antibiotics and improper stewardship being among the common causes ${ }^{3}$. In addition, overuse and inappropriate use of antimicrobial agents has been well described to pray an important role in emergence and spread of antimicrobial resistance (AMR) ${ }^{4}$. Ability to develop antimicrobial tolerance and resistance with propagation of resistance clones has resulted into therapeutic failure consequently leading to increased mortality and morbidity ${ }^{5}$. Wounds are highly susceptible to colonization and infection, which has posed a major challenge in healing that often results into death of the victims. In hospital or Health care settings, the challenge remains worse due to nosocomial bacterial infection often characterized by multidrug resistance ${ }^{6}$. Infected wounds are often associated with delayed epidermal maturation and deep scar formation that results into prolonged hospitalization and high mortality rate due to sepsis. Bacteria 
pathogens are the main etiological agents of wound infection in the community and hospital settings ${ }^{7}$. These bacterial agents are increasingly resistant to commonly used antibiotics leading to emergency of cefoTaXime (CTX-M beta-lactamase enzymes) in Klebsiella species and Methicillin Resistant S. aureus (MRSA) infections ${ }^{8}$. The outcome of wounds infected with antimicrobial resistant bacteria is often poor characterized with prolonged debility and increased cost of healthcare to the patients. Therefore, awareness of bacterial pathogens in the wounds, management standards and prevailing susceptibility patterns is a prerequisite for the rational antimicrobials use. Data on profile of clinical significant bacteria and their respective antibiotic drug resistance in Uganda is still limited. In this study we emphasized on phenotypic characterization of bacteria that cause wound infections at Kabale Regional Hospital (KRRH) and determining the drug susceptibility profiles of those agents.

\subsection{Materials And Methods}

\subsection{Study design}

This was a descriptive cross sectional study and it was conducted on patients presenting with wounds at Kabale Regional Referral Hospital (KRRH).

\subsection{Sample Collection and Transport}

Pus (Swabs and aspirates) were collected from wounds (traumatic wounds and surgical wounds) during routine clinical work from the period of June 2016 - to June 2017. Sterile cotton swabs (ThermoFisher ${ }^{\mathrm{TM}}$ ) were used to collect specimens which were immediately taken to the microbiology laboratory for microbiological investigations.

\subsection{Isolation and Identification of Microorganisms}

After accession into Laboratory register, the clinical samples were directly inoculated onto Blood agar (BAP), MacConkey agar (Oxoid ${ }^{\mathrm{TM}}$ ) and Chocolate agar (Choc). Both BAP and MacConkey agar were incubated aerobically and while Choc plate was incubated in $5-10 \% \mathrm{CO} 2$. All culture plates were incubated under humid condition of $55-70 \%$ at temperature of $35-37^{\circ} \mathrm{C}$ for $24-72$ hours with regular inspection every after 24 hours for bacterial growth. Bacterial colonial growth was identified phenotypically through macroscopic appearance, gram staining and biochemical identification using analytical profile index - API (BioMérieux $\left.{ }^{\mathrm{TM}}\right)^{9}$. On addition to API, API staph was used to confirm staphylococcus species after identification with catalase, coagulase and Staphaurex agglutination test $\left(\right.$ Remel) ${ }^{10}$.

\subsection{Antibiotic Susceptibility Testing}

Kirby-Bauer technique using Mueller Hinton Agar (Oxoid ${ }^{\mathrm{TM}}$ ) was used for antibiotic susceptibility testing (AST) following the Clinical Laboratory Standards Institute $(\mathrm{CLSI})^{11}$. The ASTs results were interpreted in accordance with the antibiotic susceptibility break-points described in the CLSI 2018) guidelines. 
Phenotypic screening of Extended Spectrum Beta Lactamase (ESBL) using double disk diffusion testing was done on MHA, using gram negative bacteria that showed phenotypic expression of ExtendedSpectrum Beta-lactamases (ESBLs). E. coli, Klebsiella spp. and Proteus mirabilis isolates displaying decreased susceptibility to ceftriaxone (CRO $30 \mu \mathrm{g}$ ) with zone diameter of $\leq 25 \mathrm{~mm}$ ) and Ceftazidime (CAZ $30 \mu \mathrm{g}$ ) with zone diameter of $\leq 22 \mathrm{~mm}$ were selected for ESBL confirmatory testing as per CLSI guidelines $^{12}$. Ceftazidime with clavulanic acid (CAZ/CLA 30/10 $\mu \mathrm{g}$ ), Ceftazidime (CAZ $30 \mu \mathrm{g}$ ), cefotaxime (CTX $30 \mu \mathrm{g}$ ), and cefotaxime with clavulanic acid (CTX/CLA 30/10 $\mu \mathrm{g}$ ) antibiotics were used for this study. Equal or greater than $5 \mathrm{~mm}$ ( $\geq 5 \mathrm{~mm}$ ) diameter zone of inhibition for the CAZ/CLA and CTX/CLA disk versus the corresponding CAZ or CTX disk was considered positive for ESBL ${ }^{13}$.

\subsection{Quality control}

Every new batch of antibiotics disks and culture media used during our study were routinely quality controlled. Media PH was adjusted using $1 \mathrm{NNaOH}$ and $1 \mathrm{NHCL}$. MacConkey agar was checked for fertility using E. coli ATCC 25922, Proteus milabilis ATCC 12453 and Enterococcus faecalis 29212, while BAP was controlled with S. aureus ATCC 25923, S. pyogenes 19615 and E. coli 25922. However, Choc was quality controlled with Haemophilus influenza ATCC 10211 and N. gonorrhaeae ATCC 43069 while MHA we used S. aureus ATCC 25923, E. coli 25922 and Pseudomonas aeruginosa ATCC $27853^{11,14}$. Results were documented in the laboratory record book and data extraction forms and reported back to clinical team for patient management. AST results were designated in terms of Sensitive (S), Intermediate (I), and Resistant (R) based on current CLSI - 2018 guidelines (9). Klebsiella pneumoniae ATCC700603 and Escherichia coli ATCC 25922 were used as ESBL-positive and ESBL-negative control strains.

\subsection{Data Management and Analysis}

Data generated were entered into Excel spread sheet, cleaned and processed to give descriptive statistics and presented as a percentage, or frequencies proportions of each isolated bacterium to determine the most prevalent species involved in wound infections and respective antimicrobial resistance patterns.

\subsection{Results}

\subsection{Bacteria isolated from patients presenting with wounds at KRRH}

Two hundred and seventy-six [ $n=276]$ wound pus swabs were cultured among which $70.7 \%(n=195)$ were culture positive with $50.8 \%(n=99)$ having a single pathogen and $49.2 \%(n=96)$ having more than one bacterial isolate. $31.79 \%(n=62)$ was $S$. aureus which was the most frequently isolated bacteria followed by $29.7 \%(n=58)$ of E. coli. In addition, $15.4 \%(n=30)$ was Klebsiella pneumoniae, $8.7 \%(n=17)$ Proteus milabilis; $3.6 \%(n=07)$ Pseudomonas aeruginosa; $3.6 \%(n=07)$ Streptococcus pyogenes; $2.0 \%(n=04)$ Enterococcus faecalis, 2.1\% $(\mathrm{n}=04)$ Citrobacter freundii, $2.1 \%(\mathrm{n}=04)$ of coagulase negative staphylococcus (CoNS) and1.0\% ( $\mathrm{n}=02)$ of Acinetobactersp. Summarized in figure 1 


\subsection{Antimicrobial resistance profile among bacteria isolated from patients presenting with wounds at KRRH}

Among $S$. aureus isolates, $90 \%(n=56)$ were resistant to trimethoprim-sulfamethoxazole and penicillin while $95 \%(n=59)$ were resistant to chloramphenicol. High resistances were also observed against tetracycline;71\% $(n=44)$ and clindamycin; $68 \%(n=42)$. However, $24 \%(n=15)$ of $S$. aureus were resistant to levofloxacin and Piperacillin-Tazobactam. There was no antimicrobial resistance against Vancomycin observed. However, 68\% $(n=42)$ were methicillin resistant(MRSA), among which $76 \%(n=32)$ were resistant to chloramphenicol, $54 \%(n=23)$ to tetracycline, $45 \%(n=19)$ against clindamycin, $29 \%(n=12)$ were resistant to Piperacillin-Tazobactam while $10 \%(n=04)$ against levofloxacin. Of note, $100 \%(n=42)$ of MRSA were resistant to trimethoprim-sulfamethoxazole (figure 2).

Among CoNS, 100( $\mathrm{n}=4)$ were resistance to Oxacillin and Ampicillin $(30 \mu \mathrm{g})$, Amoxicillin - clavulanic acid $(30 \mu \mathrm{g})$, Trimethoprim - sulfamethoxazole $(23.75 \mu \mathrm{g} / 1.25 \mu \mathrm{g})$ and Chloramphenicol(30 $\mu \mathrm{g})$. However, $50 \%$ $(n=02)$ of the isolates were resistant to Gentamycin $(10 \mu \mathrm{g})$, Ciprofloxacin $(5 \mu \mathrm{g})$, Clindamycin $(2 \mu \mathrm{g})$, Piperacillin-Tazobactam100/10 $\mu \mathrm{g})$ and Tetracycline $(30 \mu \mathrm{g})$ as shown in the figure 3

Among the S. pyogenes isolates $(n=4)$ there no resistance against Levofloxacin and Vancomycin, while $25 \%(n=1)$ were resistance to penicillin, Ampicillin and Piperacillin-Tazobactam, Clindamycin, Gentamycin and amoxicillin- clavulanic acid. However, 50\%(n =2) were resistant to Trimethoprim sulfamethoxazole, chloramphenicol, Tetracycline and Ciprofloxacin(Table 1). Among the Enterococcus (n $=4), 100 \%(n=4)$ resistant to penicillin, Ampicillin and Piperacillin-Tazobactam, Clindamycin, Trimethoprim - sulfamethoxazole, and amoxicillin- clavulanic acid. However, $75 \%(n=3)$ were resistance to Gentamycin, Levofloxacin, chloramphenicol, Tetracycline and Ciprofloxacin. There was no resistance against Vancomycin(Table 1).

Table 1: Antibiotic resistance profile of isolated streptococcus pyogenes and enterococcus species

\begin{tabular}{|c|c|c|}
\hline $\begin{array}{l}\text { Antibiotics } \\
\left(\text { OXOID }^{\mathrm{TM}}\right)\end{array}$ & $\begin{array}{l}\text { Pyogenes } \\
(n=4)\end{array}$ & $\begin{array}{l}\text { Enterococcus } \\
(n=4)\end{array}$ \\
\hline $\mathrm{AP}(30 \mathrm{ug})$ & $01(25 \%)$ & $04(100 \%)$ \\
\hline AMC(30ug) & $01(25 \%)$ & $04(100 \%)$ \\
\hline CN(10ug) & $01(25 \%)$ & 03(75\%) \\
\hline $\operatorname{SXT}(23.75 \mu \mathrm{g} / 1.25 \mu \mathrm{g} \mathrm{ug})$ & $02(50 \%)$ & $04(100 \%)$ \\
\hline CIP(5ug) & $02(50 \%)$ & 03(75\%) \\
\hline C(10ug) & $02(50 \%)$ & $03(75 \%)$ \\
\hline Te(30ug) & $02(50 \%)$ & $03(75 \%)$ \\
\hline CD(2ug) & $01(25 \%)$ & $04(100 \%)$ \\
\hline V(30ug) & 0 & 0 \\
\hline Lev(5ug) & 0 & $03(75 \%)$ \\
\hline 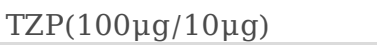 & $01(25 \%)$ & $04(100 \%)$ \\
\hline $\mathrm{P}(30 \mu \mathrm{g})$ & $01(25 \%)$ & $04(100 \%)$ \\
\hline
\end{tabular}

Footnote: $C=$ chloramphenicol, $T e=$ Tetracycline $C D=$ Clindamycin, $V=$ Vancomycin, Lev $=$ Levofloxacin, $T Z P=$ PiperacillinTazobactam, AP = Ampicillin, AMC = Amoxicillin - clavulanic acid, $C N=$ Gentamycin, $S X T=$ Trimethoprim - sulfamethoxazole, $C I F$ 
Among the Enterobacteriaceae isolated ( $n=99)$, their antimicrobial resistant patterns to commonly used antibiotics (table 2) were varying indicating that both isolates were $100 \%$ resistant to Ampicillin. In addition, $86 \%(\mathrm{n}=50)$ E. coli, $100 \%(\mathrm{n}=30)$ Klebsiella sp. $100 \%(\mathrm{n}=7)$ Proteus sp. and $100 \%(\mathrm{n}=4$ Citrobactersp. were resistant to Amoxicillin- clavulanic acid.

More to that, $48 \%(\mathrm{n}=28)$ E. coli, $57 \%(\mathrm{n}=4)$ Proteus sp., $100 \%(\mathrm{n}=30)$ Klebsiella sp., and $50 \%(\mathrm{n}=2)$ Citrobacter $s p$. were resistant to gentamycin while $83 \%(\mathrm{n}=48)$ E. coli, $100 \%(\mathrm{n}=30)$ Klebsiella $s p ., 43 \%(\mathrm{n}$ = 3) Proteus sp., and 50\% ( $n=2)$ Citrobacter sp. were resistant to Cefuroxime. However, $100 \%$ of E. coli, Klebsiella sp., Proteus sp. and 75\% $(n=3)$ Citrobacter $s p$. were resistant to Trimethoprim sulfamethoxazole while $48 \%(\mathrm{n}=28)$ of E. coli, $93 \%(n=28)$ Klebsiella $s p ., 71 \%(n=5)$ Proteus sp. and $75 \%$ $(n=3)$ Citrobacter $s p$. were resistant to ciprofloxacin. In addition, $83 \%(n=48)$ of E. coli, $100 \%(n=30)$ Klebsiella sp.,71\% $(n=5)$ Proteus sp. and 50\% $(n=2)$ Citrobacter sp. were resistant to chloramphenicol. Of note, $5 \%(n=3)$ of E. coli, $17 \%(n=5)$ Klebsiella sp., $14 \%(n=2)$ Proteus $s p$. and $50 \%(n=2)$ Citrobacter $s p$. were resistant to Imipenem while $86 \%(\mathrm{n}=50)$ of E. coli, $50 \%(n=15)$ Klebsiella $s p ., 71 \%(n=5)$ Proteus sp. and 50\% (n=2) Citrobacter sp. were resistant to Tetracycline. We also observed that, $100 \%(n=58)$ of E. coli, 93\% $(n=28)$ Klebsiella sp.,57\% $(n=4)$ Proteus sp. and 75\% $(n=3)$ Citrobacter $s p$. were resistant to erythromycin while $34 \%(\mathrm{n}=20)$ of E. coli, $90 \%(n=27)$ Klebsiella sp.,57\% $(n=4)$ Proteus sp. and $75 \%(n=$ 3) of Citrobacter $s p$. were resistant to ceftriaxone. We also report that, $14 \%(\mathrm{n}=8)$ of E. coli, $100 \%(n=30)$ Klebsiella sp.,57\% $(n=4)$ Proteus sp. were resistant to Cefixime (CFM) while $86 \%(n=50)$ of E. coli, $70 \%(n$ = 21) Klebsiella $s p ., 57 \%(n=4)$ Proteus $s p$. and $25 \%(n=1)$ Citrobacter $s p$. were resistant to Cefpodoxime (CPD). In addition, $57 \%(\mathrm{n}=33)$ of E. coli, $67 \%(n=20)$ Klebsiella sp., $71 \%(n=5)$ Proteus sp. and $50 \%(n=$ 2) Citrobacter $s p$. were resistant cefotaxime (CTX) while 34\% $(\mathrm{n}=20)$ of E. coli, $90 \%(n=27)$ Klebsiella $s p ., 57 \%(n=4)$ Proteus sp. and 25\% $(n=1)$ Citrobacter $s p$. were resistant Cefepime (CFEP).

Table 2: antibiotic resistance profile of isolated Enterobacteriaceae bacteria 


\begin{tabular}{|c|c|c|c|c|}
\hline Antibiotics (Oxoid ${ }^{\mathrm{TM}}$ & $\begin{array}{l}\text { E. coli } \\
(n=58)\end{array}$ & $\begin{array}{l}\text { Klebsiella } \\
(\mathrm{n}=30)\end{array}$ & $\begin{array}{l}\text { p.Proteus sp. } \\
(n=7)\end{array}$ & $\begin{array}{l}\text { Citrobacter sp. } \\
(n=4)\end{array}$ \\
\hline AP(30ug) & $58(100 \%)$ & $30(100 \%)$ & 07(100\%) & 04(100\%) \\
\hline AMC(30ug) & $50(86 \%)$ & $30(100 \%)$ & $07(100 \%)$ & $04(100 \%)$ \\
\hline CN(10ug) & $28(48 \%)$ & $30(100 \%)$ & $04(57 \%)$ & $02(50 \%)$ \\
\hline CXM(30ug) & 48(83\%) & $30(100 \%)$ & 03(43\%) & $02(50 \%)$ \\
\hline SXT(25ug) & $58(100 \%)$ & $30(100 \%)$ & 07(100\%) & 03(75\%) \\
\hline CIP(5ug) & $28(48 \%)$ & $28(93 \%)$ & $05(71 \%)$ & 03(75\%) \\
\hline $\mathrm{C}(10 \mathrm{ug})$ & 48(83\%) & $30(100 \%)$ & $05(71 \%)$ & $02(50 \%)$ \\
\hline IMI(10ug) & $03(5 \%)$ & $05(17 \%)$ & $02(14 \%)$ & $02(50 \%)$ \\
\hline Te(30ug) & $50(86 \%)$ & $15(50 \%)$ & $05(71 \%)$ & $02(50 \%)$ \\
\hline E(15ug) & $58(100 \%)$ & $28(93 \%)$ & $4(57 \%)$ & $03(75 \%)$ \\
\hline CRO3(30ug) & $20(34 \%)$ & $27(90 \%)$ & $4(57 \%)$ & $01(25 \%)$ \\
\hline CEF(30ug) & $08(14 \%)$ & $30(100 \%)$ & $4(57 \%)$ & 0 \\
\hline CPD(10 $\mu \mathrm{g})$ & $50(86 \%)$ & $21(70 \%)$ & $4(57 \%)$ & $01(25 \%)$ \\
\hline CTX(30ug) & $33(57 \%)$ & $20(67 \%)$ & $05(71 \%)$ & $02(50 \%)$ \\
\hline CFEP(30ug) & $20(34 \%)$ & $27(90 \%)$ & $4(57 \%)$ & $01(25 \%)$ \\
\hline
\end{tabular}

Footnote: $I M I=$ Imipenem, Te =Tetracycline =Erythromycin, $C D=$ Clindamycin, $C R O=C e f t r i a x o n e, C E F=C e f i x i m e C P D=$ Cefpodoxime, $C T X=$ cefotaxime,$C F E P=$ Cefepime,$A P=$ Ampicillin, $A M C=$ Amoxicillin - clavulanic acid, $C N=G e n t a m y c i n, S X T=$ Trimethoprim - sulfamethoxazole, CIP = Ciprofloxacin, $P=$ penicillin.

Among the non-enteric gram negative bacteria isolated $(n=9)$ were $P$. aeruginosa $(n=7)$ and Acinetobacter sp. $(n=2)$. Among which $100 \%(n=7)$ of $P$. aeruginosa isolates were resistant to chloramphenicol, ceftriaxone, Cefpodoxime and cefotaxime. However, resistance was observed Piperacillin-Tazobactam; $14 \%(n=1)$, Ceftazidime; $100 \%(n=7)$, Cefepime; $57 \%(n=4)$, gentamicin; $57 \%(n$ $=4)$, levofloxacin; $43 \%(n=3)$, Imipenem; $29 \%(n=2)$. We noted that $50 \%(n=1)$ of the Acinetobacter $s p$. were resistant to gentamycin, chloramphenicol ceftriaxone levofloxacin cefotaxime PiperacillinTazobactam. However, all the Acinetobacter sp. isolates were not resistant to Imipenem, Tetracycline, Cefpodoxime, cefotaxime, Cefepime and Cefixime as tabulated in table 3.

Table 3: antibiotic resistance profile of $P$. aeruginosa and Acinetobacter sp.

\begin{tabular}{|c|c|c|}
\hline Antibiotics & P.aeruginosa $(\mathrm{n}=7)$ & Acinetobacter $\operatorname{sp}(n=2$ \\
\hline $\mathrm{CN}(10 \mu \mathrm{g})$ & $01(14 \%)$ & $01(50 \%)$ \\
\hline$C(10 \mu \mathrm{g})$ & $07(100 \%)$ & $01(50 \%)$ \\
\hline IMI $(10 \mu \mathrm{g})$ & $02(29 \%)$ & 00 \\
\hline TE $(30 \mu \mathrm{g})$ & $04(57 \%)$ & 00 \\
\hline CRO $(30 \mu \mathrm{g})$ & $07(100 \%)$ & $01(50 \%)$ \\
\hline CFEP $(30 \mu \mathrm{g})$ & $03(43 \%)$ & 00 \\
\hline CEF $(30 \mu g)$ & $01(14 \%)$ & 00 \\
\hline $\operatorname{Lev}(5 g)$ & $3(43 \%)$ & $01(50 \%$ \\
\hline $\mathrm{CPD}(10 \mu \mathrm{g})$ & $07(100 \%)$ & 00 \\
\hline CTX(30 $\mu \mathrm{g})$ & $07(100 \%)$ & $01(50 \%)$ \\
\hline $\mathrm{TZP}(100 \mu \mathrm{g} / 1$ & $01(14 \%)$ & $01(50 \%)$ \\
\hline
\end{tabular}

Footnote: $C=$ Chloramphenicol, $I M I=$ Imipenem, Te =Tetracycline $=$ Erythromycin, $C D=C l i n d a m y c i n, C R O=C e f t r i a x o n e, C E F=$ Cefixime $C P D=$ Cefpodoxime, $C T X=$ cefotaxime, CFEP = Cefepime, $C N=$ Gentamycin, CIP = Ciprofloxacin, TZP = Piperacillin- 


\subsection{Discussion}

Wound infections is a common occurrence among patients visiting Ugandan hospital and despite the scarcity of documented reports or information describing the profile and antibiotic susceptibility pattern of pathogenic bacteria behind these infections, they are commonly due to either surgical or injury related to trauma, accidents, or burns. Currently, wound infections are often characterized delays in health due to infection with highly virulent and antimicrobial resistant bacterial pathogens that pose a threat to treatment ${ }^{[15]}$. The increase of antimicrobial resistance among pathogenic bacteria among the inpatients and the community threatens the current management of wound infections ${ }^{[16]}$.

The majority of wound isolates were gram negative bacilli (61\%) which is in agreement with Hauke et al. from Ghana, Mekonnen et al. from Ethiopia, and Valentine et al. from Ukrainian military hospital who reported Gram negative bacteria as the predominating pathogens in wound infections ${ }^{[17,18,19]}$. Escherichia coli, Klebsiella spp., Proteus sp. and Pseudomonas spp. were other common gram negative bacteria observed in our study. In a recent report from Ukraine, Gram negative bacteria, particularly Enterobacteriaceae, were found as major pathogens ${ }^{[19]}$. However, the presence of gram positive bacteria $(39 \%)$ in the current study is supported by several literatures ${ }^{[20,7,15]}$. Staphylococcus aureus $(49.2 \%)$ was the predominant isolate responsible for bacterial wound infections in this study which is quite similar to several previous studies ${ }^{[21,6,22]}$. We confirm that the as previously described, these bacteria are traditional most prevalent microorganisms found in wound infections. Though, Gram negative bacteria have been described to be associated with hospital acquired infections and common in abdominal surgical procedures ${ }^{[23,24]}$. S. aureus and Gram negative bacterial pathogens are implicated to be common bacteria that invades and colonize wounds and are known to produce compelling virulence factors that are highly destructive with consequent delays in wound healing process while sustaining infection ${ }^{[25]}$.

Of note, we observed presence of more than one bacterial pathogens from one wound source, suggestive of Polymicrobial wound infection. This is always associated with poor wound management coupled with increased microbial survival and antimicrobial resistance. In addition, the complacence towards benefits of wound debridement and wound irrigation without antimicrobials and close wound monitoring are probable cause of multiple bacterial invasion and colonization. Infection control and prevention prays a key role wound management and it has been recommended that antimicrobials should not be prescribed as the initial treatment strategy ${ }^{[26]}$. Nevertheless, we suggest antimicrobial use as prophylaxis in case of surgical wounds, while the use of systemic antibacterial be indicated when sepsis appears and is spreading to the subcutaneous soft tissues. This can result into bacteremia with serious adverse patient outcome $^{[26,18]}$. However, longer period of prophylactic antimicrobial exposure in surgical interventions may subsidize to organisms developing resistance fast. Therefore, antimicrobials are required to eliminate microbial colonization and spread ${ }^{17}$. Current findings indicate the presence of highly drug 
resistant bacterial pathogens in wound infections. The high use of antibiotics and inadequate infection control processes in both community and hospital settings are probable cause of increasing rates of antimicrobial resistance ${ }^{18}$. We are concerned among Gram positive bacteria, presence of Methicillin Resistant Staphylococcus aureus(MRSA) in the current study which is the most resistant microbial agent. The isolates were highly resistant to Chloramphenicol, Tetracycline, trimethoprim - sulfamethoxazole, Piperacillin - Tazobactam, and clindamycin. This finding is in agreement with the previous reports of Bhat et al., Anguzu et al., and Jean-Marie et al. ${ }^{[7,27,21]}$. However, comparable to other studies in Kinshasa (Democratic republic of Congo -DRC) and India, all the isolates of CONS were identified and highly resistant to Ampicillin, amoxicillin - clavunate, trimethoprim - sulfamethoxazole and chloramphenicol[21, 20]. Trimethoprim - sulfamethoxazole is one of the most widely used antimicrobial agents for treating pyogenic wound infections, was found not susceptible to all isolates. Pressure due to continuous use of this particularly antibiotics in HIV - AIDS patients as prophylactic agent is the probable cause of resistance. S. pyogenes resistance to ciprofloxacin, trimethoprim - sulfamethoxazole, chloramphenicol and Tetracycline threatens the current treatment choice to this pathogen. Similar trends have been reported elsewhere and probable over use of these antimicrobial agents and poor wound management maybe implicated as the drivers ${ }^{[28]}$. In addition, S. pyogenes was susceptible to ampicillin, amoxicillin clavunate, Gentamycin, levofloxacin, Piperacillin - Tazobactam, Penicillin, which are one of the most widely used antimicrobial agents ${ }^{[26]}$. However, Enterococcus spp., isolates were highly resistant to ampicillin, amoxicillin - clavunate, Gentamycin, levofloxacin, Piperacillin - Tazobactam, Penicillin, ciprofloxacin, trimethoprim - sulfamethoxazole, chloramphenicol and Tetracycline, and remarkable susceptible to vancomycin. It is not a surprise to our study that enterococci sp., are known to be multidrug resistant opportunistic bacteria that become pathogenic when they colonize niches uncommon to them like wounds. They have turn out to be major cause of nosocomial infections especially surgical sites among other sites ${ }^{[29]}$. Alongside, our findings indicate the high incidence of drug resistance among Gram negative isolates too. In this study, Escherichia coli, Klebsiella spp., Proteus spp., and Citrobacter spp., were highly resistant to cephalosporins and other commonly used antibiotics while Pseudomonas aeruginosa a gram negative non-fermenter was resistant to fluoroquinolones, aminoglycosides, and cephalosporins and Penicillins used in the current study. Such susceptibility pattern of these isolates is in agreement with other previous reports across Africa ${ }^{[30]}$. Multidrug resistance among Gram negative bacteria resistant to commonly used antimicrobials in wound infections is a serious concern ${ }^{[31,17]}$. The isolated Gram negative bacteria are known to be normal flora of the gut. To minimize the selection pressure of individual antibacterial on the normal flora, we advise use of narrow-spectrum agents. Empirical treatment should be discouraged rather base on the wound culture results. Acinetobacter sp., isolated was not highly resistant to commonly used antimicrobials. However, this particular pathogen is important nosocomial bacteria often associated with a wide range of beta-lactam resistant and extended spectrum beta lactam resistance with ability to produce biofilms, among other characteristics, allows it to persist in hospitals for prolonged periods $\left.{ }^{[32} 33\right]$.

\section{Conclusions}


Wound infections were mainly caused by gram negative bacteria particularly, Escherichia coli, Klebsiella spp., Proteus spp., and Pseudomonas spp. As well as gram positive cocci particularly S. aureus. There were high levels of antimicrobial resistance among both Gram negative and Gram positive bacteria. Continuous surveillance is obligatory to appraise and inform on bacterial and their respective antimicrobial susceptibility profiles in wound isolates. This is essential for provision of suitable antimicrobial agent in the treatment of wound infections and prevention of antimicrobial resistance.

\section{Transparency Declarations}

None to declare.

\section{Abbreviations}

AMR

Antimicrobial Resistance

AST

Antibiotic Susceptibility Test

BAP

Blood Agar Plate

Choc

Chocolate agar

CLSI

Clinical and Laboratory Standard Institute

CoNS

Coagulase Negative Staphylococcus

CPHL

Central Public Health Laboratories

ESBL

Extended Spectrum Beta Lactamase

$\mathrm{KRRH}$

Kabale Regional Referral Hospital

\section{Declarations}

\section{Availability of Data and materials}

The datasets used and/or analysed during the present study are available from the corresponding author on reasonable request.

\section{Acknowledgements}


We thank the Kabale Regional Referral Hospital Laboratory management and entire staff for providing materials and reagents that was used to collect data from clinical specimens this present research.

\section{Ethical approval and consent to participate}

This study was approved by Mbarara University of Science and Technology (MUST) Research and Ethical Committee, Uganda National Council of Science and Technology (UNCST) study Number 13/08-15. The need for informed consents was waived owing to the infection control nature of this study.

\section{Funding}

This research was supported by Kabale Regional Referral Hospital (KRRH) in providing Laboratory space, reagents and materials. KRRH had no role in the design of the study and collection, analysis, and interpretation of data and in writing the manuscript.

\section{Author information}

\section{Author notes}

Andrew Baguma, Benson Musinguzi, Atek Kagirita, Joel Bazira contributed equally to this work.

\section{Affiliations}

Department of Biomedical Sciences, Kabale University School of Medicine(KABSOM),Kabale University.

Andrew Baguma (AB)

Department of Medical Laboratory Sciences, School of Allied Health Sciences, Kampala International University

Benson Musinguzi (BM)

Central Public Health Laboratories (CPHL), Ministry of Health, Uganda.

Atek Kagirita(AK)

Department of Microbiology, Faculty of medicine, Mbarara University of Science and Technology.

Joel Bazira (JB)

\section{Contributions}

$A B$ and $B M$ analysed and interpreted the data and drafted the manuscript. JB and $A B$ designed the study, collected the sample and analyse the data. AK coordinated the data preparation, supervised the data analysis and revised the manuscript. The authors read and approved the final manuscript. 


\section{Corresponding authors}

Correspondence to Andrew Baguma

\section{Ethics declarations}

\section{Consent for publication}

Not applicable.

\section{Competing interests}

The authors declare that they have no competing interests.

\section{References}

1. Stanley, I. \& Bwanga, F. and Antibiotic Susceptibility Patterns of Clinical Isolates of MethicillinResistant Staphylococcus aureus in a Tertiary Care Hospital in Western Uganda. Br. Microbiol. ... (2014).

2. Brennan, G. I. et al. Emergence of hospital- and community-associated panton-valentine leukocidinpositive methicillin-resistant Staphylococcus aureus genotype ST772-MRSA-V in Ireland and detailed investigation of an ST772-MRSA-V cluster in a neonatal intensive care unit. J. Clin. Microbiol. 50, 841-7 (2012).

3. Sapula, S. A. \& Brown, M. H. Antimicrobial Drug Efflux Pumps in Staphylococcus aureus. in EffluxMediated Antimicrobial Resistance in Bacteria 165-195 (Springer, 2016).

4. Davies, J. \& Davies, D. Origins and Evolution of Antibiotic Resistance. Microbiol. Mol. Biol. Rev. 74, 417-433 (2010).

5. Blanco, P. et al. Bacterial Multidrug Efflux Pumps: Much More Than Antibiotic Resistance Determinants. Microorganisms 4, 14 (2016).

6. Yasidi, B. \& Akawu, D. Retrospective Analysis of Bacterial Pathogens Isolated from Wound Infections at a Tertiary Hospital in Nguru, Yobe State Nigeria. Am. J. ... (2015).

7. Bhat, V. \& Vasaikar, S. Bacteriological profile and antibiogram of aerobic burn wound isolates in Mthatha, Eastern Cape, South Africa. South Afr J Epidemiol Infect Peer Rev. SAJEI South Afr J Epidemiol Infect 2525, 2010-5 (2010).

8. Ogefere, H. O., Aigbiremwen, P. A. \& Omoregie, R. Extended-Spectrum Beta-Lactamase ( ESBL )Producing Gram-negative Isolates from Urine and Wound Specimens in a Tertiary Health Facility in Southern Nigeria. 14, 1089-1094 (2015).

9. CLSI, 2015. M100-S25 Performance Standards for Antimicrobial. (2015).

10. Kloos, W. E. \& Wolfshohl, J. F. Identification of Staphylococcus species with the API STAPH-IDENT system. J. Clin. Microbiol. 16, 509-516 (1982). 
11. CLSI, 2015. M07-A10: Methods for Dilution Antimicrobial Susceptibility Tests for Bacteria That Grow Aerobically; Approved Standard-Tenth Edition. 7-58 (2015).

12. Poulou, A. et al. Modified CLSI extended-spectrum??-lactamase (ESBL) confirmatory test for phenotypic detection of ESBLs among Enterobacteriaceae producing various??-lactamases. J. Clin. Microbiol. 52, 1483-1489 (2014).

13. Shaikh, S., Fatima, J., Shakil, S., Rizvi, S. M. D. \& Kamal, M. A. Antibiotic resistance and extended spectrum beta-lactamases: Types, epidemiology and treatment. Saudi J. Biol. Sci. 22, 90-101 (2015).

14. Dejulius, K. L. et al. Use of BBL ${ }^{\mathrm{TM}}{ }^{\mathrm{CHROM}}$ Magar ${ }^{\mathrm{TM}}$ Orientation Media for the Identification and Enumeration of Urinary Tract Pathogens: Comparison to Routine Culture Techniques BY SPECIFIC BACTERIAL ENZYME. (2004).

15. Kaup, S. \& Sankarankutty, J. Prevalence and antimicrobial susceptibility patterns of bacteria isolated from skin and wound infections. 4, 39-45 (2014).

16. Omole, I. \& Stephen, E. ANTIBIOGRAM PROFILE OF BACTERIA ISOLATED FROM WOUND INFECTION OF PATIENTS IN THREE HOSPITALS IN ANYIGBA, KOGI STATE, NIGERIA. FUTA J. Res. ... (2015).

17. Wounds, I. et al. Antimicrobial-Resistant Bacteria in Infected Wounds, Ghana, 2014 1. 24, 2016-2019 (2018).

18. Sisay, M., Worku, T. \& Edessa, D. Microbial epidemiology and antimicrobial resistance patterns of wound infection in Ethiopia: a meta-analysis of laboratory-based cross-sectional studies. 9, 1-19 (2019).

19. Valentine, K. P. \& Viacheslav, K. M. Bacterial flora of combat wounds from eastern Ukraine and time specified changes of bacterial recovery during treatment in Ukrainian military hospital. 1-7 (2017) doi:10.1186/s13104-017-2481-4.

20. Mythri, B. A., Patil, A. B., Arati, K. \& Sharon, V. A. Aerobic Bacteriological Profile from Wound Site Infections in Road Traffic Accident ( RTA ) Patients. Indian J Microbiol Res 3, 37-39 (2016).

21. Jean-Marie Liesse lyamba1,\&, José Mulwahali Wambale1, Cyprien Mbundu Lukukula1, N. za B. T.-K. \& 1 Laboratory. High prevalence of methicillin resistant staphylococci strains isolated from surgical site infections in Kinshasa. Pan African Med. Journa; 8688, 1-7 (2014).

22. Vincze, S. et al. Alarming proportions of methicillin-resistant Staphylococcus aureus (MRSA) in wound samples from companion animals, Germany 2010-2012. PLoS One 9, (2014).

23. Mehrad, B., Clark, N. M., Zhanel, G. G. \& Lynch, J. P. Antimicrobial Resistance in Hospital-Acquired Gram-Negative Bacterial Infections. Chest 147, 1413-1421 (2015).

24. Mehrad, B. \& Clark, N. Antimicrobial Resistance in Hospital-Acquired Gram-Negative Bacterial Infections. CHEST ... (2015).

25. Wong, S. Y., Manikam, R. \& Muniandy, S. Original Article Prevalence and antibiotic susceptibility of bacteria from acute and chronic wounds in Malaysian subjects. $J$ Infec Dev Ctries 9, 936-944 (2015). 
26. Bowler, P. G. \& Duerden, B. I. Wound Microbiology and Associated Approaches to Wound Management. 14, 244-269 (2001).

27. Anguzu, J. R. \& Olila, D. Drug sensitivity patterns of bacterial isolates from septic post-operative wounds in a regional referral hospital in Uganda. Afr. Health Sci. 7, 148-154 (2007).

28. Fernandes, P. B. et al. New Macrolides Active against Streptococcus pyogenes with Inducible or Constitutive Type of Macrolide-Lincosamide- Streptogramin B Resistance. 33, 78-81 (1989).

29. Olawale, K. O., Fadiora, S. O. \& Taiwo, S. S. Prevalence of Hospital-Acquired Enterococci Infections in Two Primary-Care Hospitals in Osogbo, Southwestern Nigeria. African J. Infect. Dis. 5, 40-46 (2011).

30. Lai, P. S., Bebell, L. M., Meney, C., Valeri, L. \& White, M. C. Epidemiology of antibiotic-resistant wound infections from six countries in Africa. 1-7 (2018) doi:10.1136/bmjgh-2017-000475.

31. Rijal, B. P., Satyal, D. \& Parajuli, N. P. High Burden of Antimicrobial Resistance among Bacteria Causing Pyogenic Wound Infections at a Tertiary Care Hospital in Kathmandu, Nepal. 2017, (2017).

32. Dahdouh, E. et al. Patterns of Biofilm Structure and Formation Kinetics among Acinetobacter baumannii Clinical Isolates with Different Antibiotic Resistance Profiles. Med. Chem. Commun. (2015) doi:10.1039/C5MD00377F.

33. Sinha, M., Srinivasa, H. \& Macaden, R. Antibiotic resistance profile \& extended spectrum betalactamase ( ESBL ) production in Acinetobacter species. 63-67 (2007).

\section{Figures}

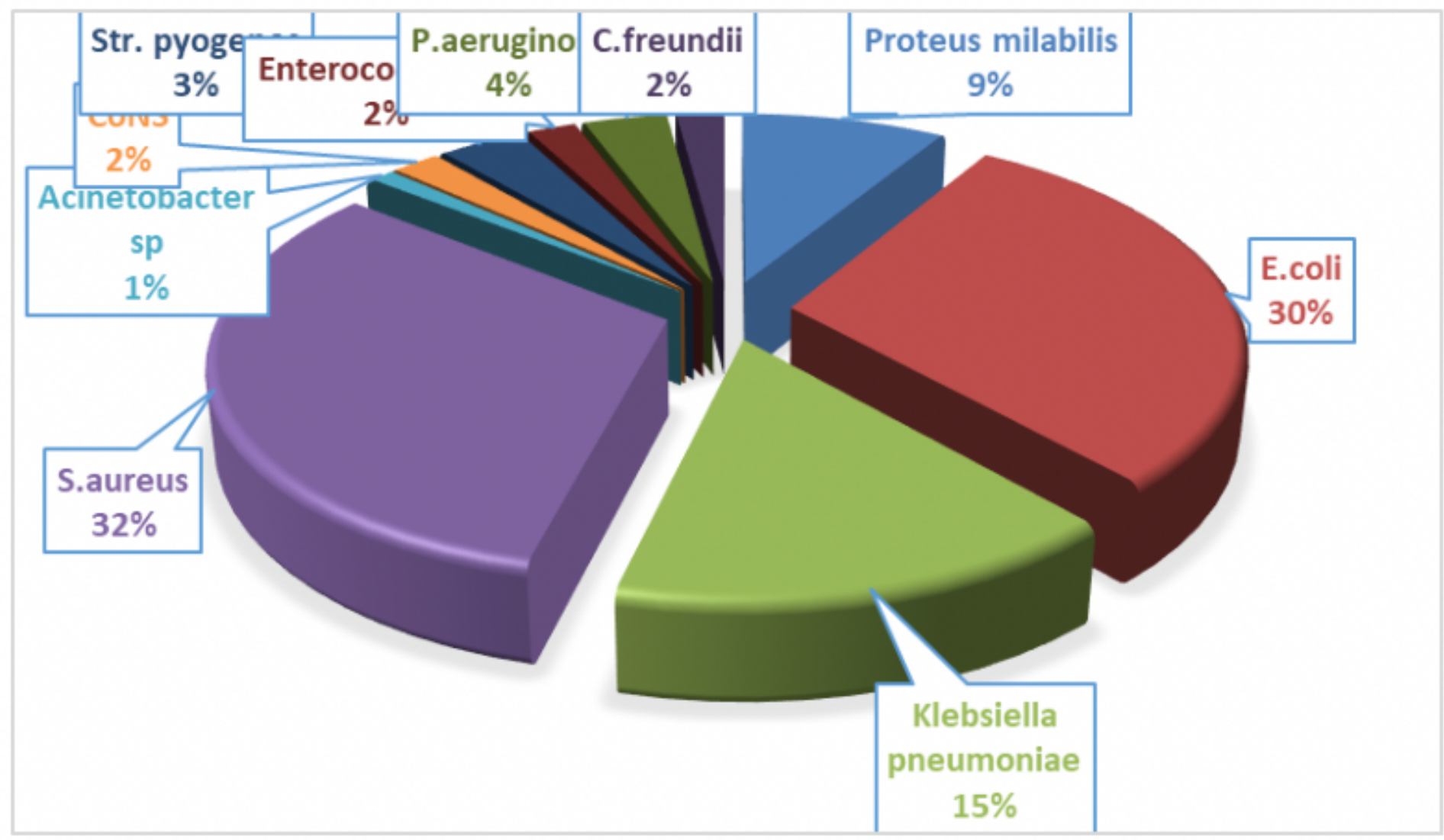


Figure 1

Isolated bacteria from clinical pus samples

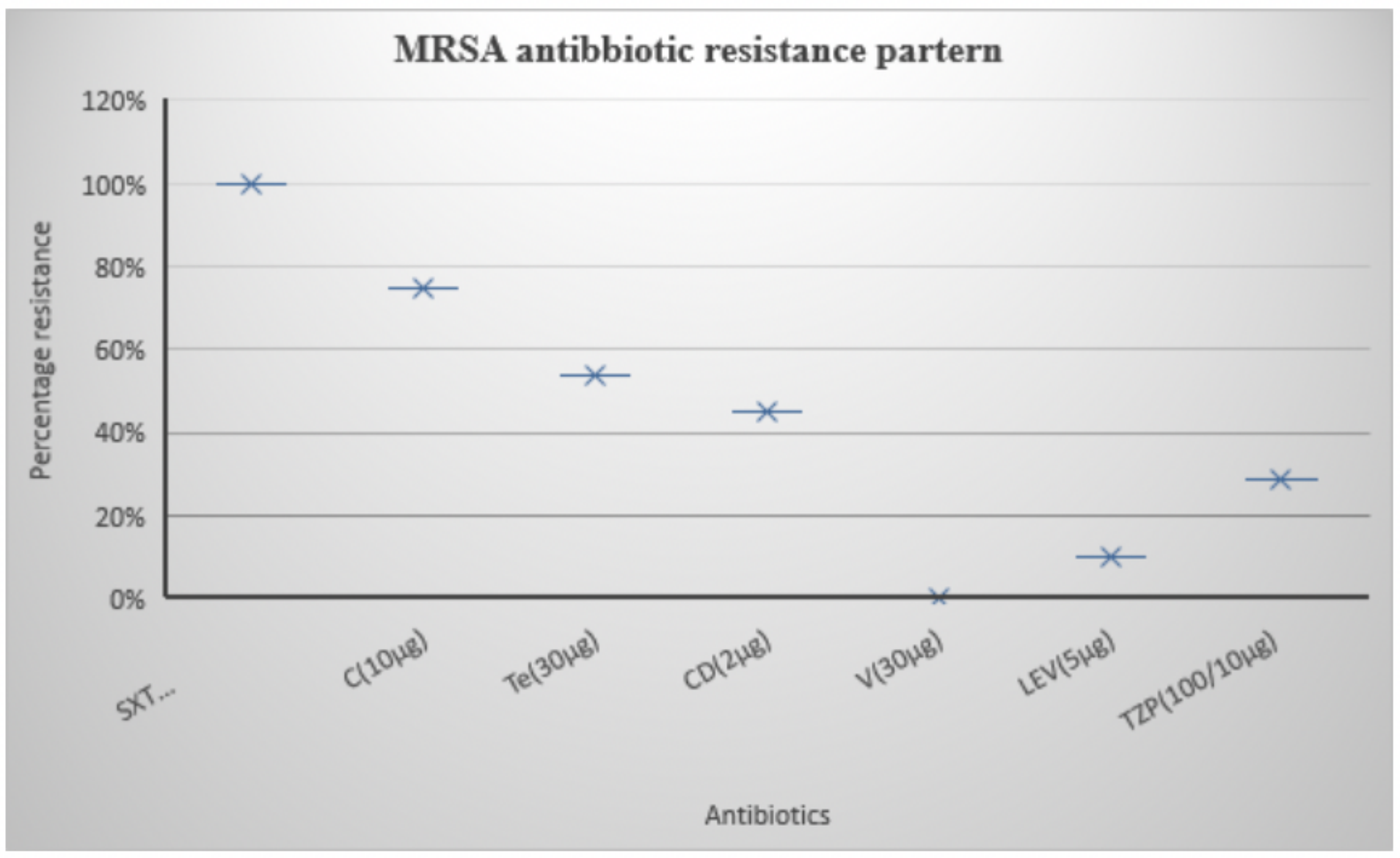

Figure 2

Antibiotic profile of MRSA to commonly used antibiotics Footnote: $\mathrm{C}=$ chloramphenicol, $\mathrm{Te}=$ Tetracycline, $\mathrm{CD}=$ Clindamycin, $\mathrm{V}=$ Vancomycin, LEV = Levofloxacin, TZP = Piperacillin-Tazobactam 


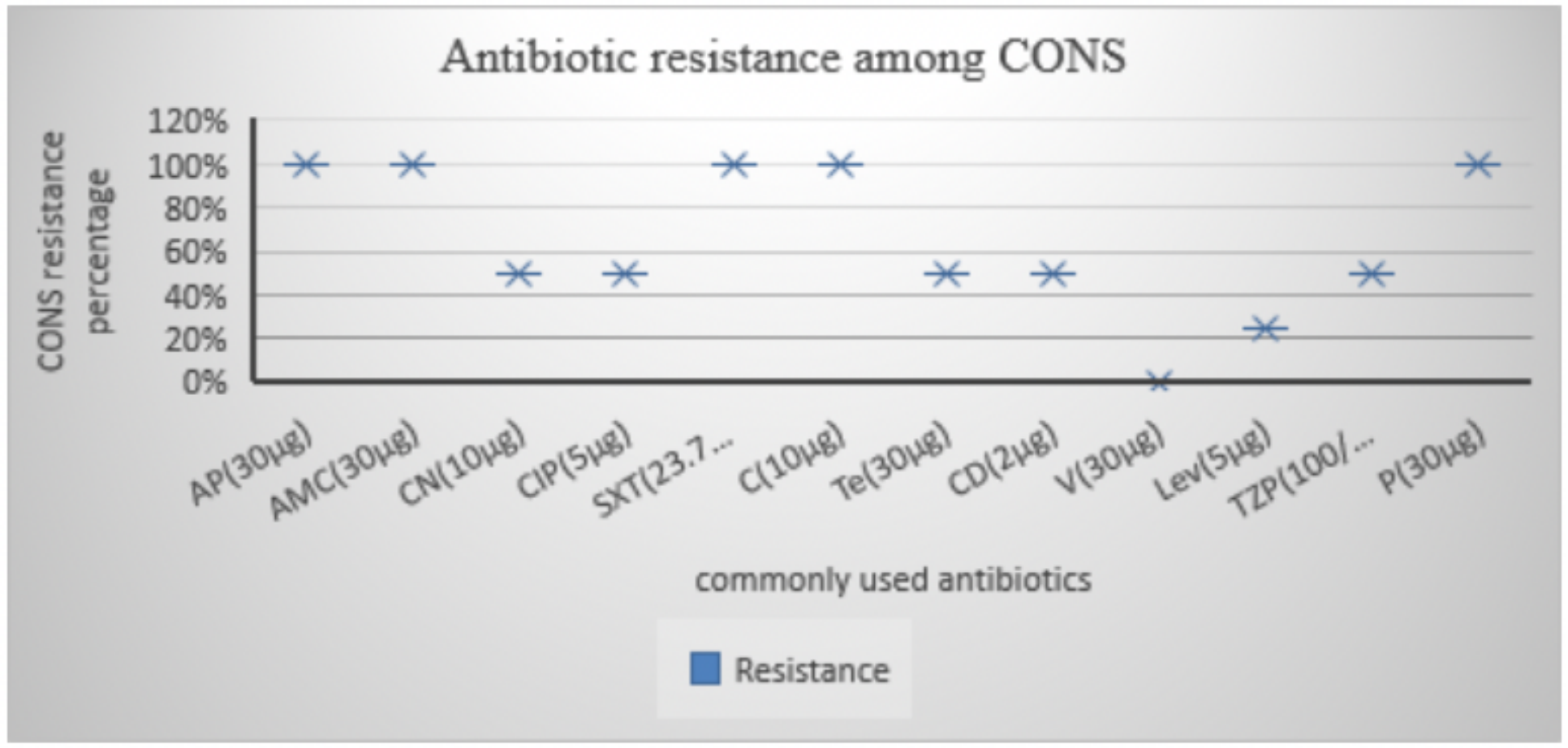

\section{Figure 3}

Antibiotic profile of MR CoNS to commonly used antibiotics Footnote: CONS = Coagulase negative staphylococcus, $\mathrm{C}=$ chloramphenicol, $\mathrm{Te}=$ Tetracycline, $\mathrm{CD}=$ Clindamycin, $\mathrm{V}=$ Vancomycin, $\mathrm{LEV}=$ Levofloxacin, TZP = Piperacillin-Tazobactam 